\title{
LIMIT CYCLES OF GENERALIZED LIÉNARD POLYNOMIAL DIFFERENTIAL SYSTEMS VIA AVERAGING THEORY
}

\author{
BELEN GARCÍA ${ }^{1}$, JAUME LLIBRE ${ }^{2}$ AND JESÚS S. PÉREZ DEL RÍO ${ }^{1}$
}

\begin{abstract}
Using the averaging theory of first and second order we study the maximum number of limit cycles of the polynomial differential systems$$
\dot{x}=y, \quad \dot{y}=-x-\varepsilon\left(p_{1}(x) y+q_{1}(x) y^{2}\right)-\varepsilon^{2}\left(p_{2}(x) y+q_{2}(x) y^{2}\right) .
$$

which bifurcate from the periodic orbits of the linear center $\dot{x}=y, \dot{y}=-x$. Here $\varepsilon$ is a small parameter. If the degrees of the polynomials $p_{1}, p_{2}, q_{1}$ and $q_{2}$ is $n$, then we prove that this maximum number is $[n / 2]$ using the averaging theory of first order, where [.] denotes the integer part function; and this maximum number is at most $n$ using the averaging theory of second order.
\end{abstract}

\section{IntroduCtion AND STATEMENT OF THE RESUlts}

The second part of the 16th Hilbert's problem asks for finding an upper bound on the maximum number of limit cycles which can have the class of all planar polynomial differential systems with a fixed degree. Since this problem up to now is untractable Smale in [34] proposed to restrict it to the class of classical Liénard differential systems of the form

$$
\dot{x}=y, \quad \dot{y}=-x-f(x) y,
$$

where $f(x)$ is a polynomial, or equivalently of the form

$$
\dot{x}=y-F(x), \quad \dot{y}=-x, \quad \text { where } F(x)=\int f(x) d x .
$$

For these systems in 1977 Lins, de Melo and Pugh [19] stated the conjecture that if $f(x)$ has degree $n \geq 1$ then system (1) has at most $[n / 2]$ limit cycles. They prove this conjecture for $n=1,2$. The conjecture for $n=3$ has been proved recently by Chengzi Li and Llibre in [20]. For $n \geq 5$ the conjecture is not true, see De Maesschalck and Dumortier [7] and Dumortier, Panazzolo and Roussarie [8]. So it remains to know if the conjecture is true or not for $n=4$.

Many of the results on the limit cycles of polynomial differential systems have been obtained by considering limit cycles which bifurcate from a single degenerate singular point (i.e., from a Hopf bifurcation), that are called small amplitude limit cycles, see for instance Lloyd [27]. There are partial results concerning the number of small amplitude limit cycles for Liénard polynomial differential systems.

Other way also very used for obtaining results on the limit cycles of polynomial differential systems is perturbing the linear center $\dot{x}=y, \dot{y}=-x$ inside the class of

2010 Mathematics Subject Classification. 34C07, 34C23, 37G15.

Key words and phrases. polynomial differential systems, limit cycles, averaging theory. 
polynomial differential systems, or inside the class of classical polynomial Liénard differential systems, i.e.

$$
\dot{x}=y, \quad \dot{y}=-x-\varepsilon f(x) y,
$$

where $\varepsilon$ is a small parameter. The limit cycles obtained in this way are sometime called medium amplitude limit cycles. Of course, the number of small or medium amplitude limit cycles gives a lower bound for the maximum number of limit cycles that a polynomial differential system can have.

There are many results concerning the number of small and medium amplitude limit cycles for the following generalized Liénard differential systems

$$
\dot{x}=y, \quad \dot{y}=-g(x)-f(x) y,
$$

where $f(x)$ and $g(x)$ are polynomials, see for instance $[2,4-6,9-13,15-21,24$, $29-34,37,38]$.

The number of medium amplitude limit cycles bifurcating from the linear center $\dot{x}=y, \dot{y}=-x$ for the following three kind of generalized polynomial Liénard differential systems

$$
\begin{array}{ll}
\dot{x}=y-g_{1}(x)-f_{1}(x) y, & \dot{y}=-x-g_{2}(x)-f_{2}(x) y \\
\dot{x}=y-g_{1}(x), & \dot{y}=-x-g_{2}(x)-f_{2}(x) y ; \\
\dot{x}=y-f_{1}(x) y, & \dot{y}=-x-g_{2}(x)-f_{2}(x) y
\end{array}
$$

where studied in the papers [24], [1] and [25, 26], respectively. Moreover, the number of medium amplitude limit cycles bifurcating from the center $\dot{x}=y^{2 p-1}$, $\dot{y}=-x^{2 q-1}$ of the polynomial differential systems

$$
\dot{x}=y^{2 p-1}, \quad \dot{y}=-x^{2 q-1}-f(x) y^{2 n-1},
$$

has been analyzed in [22].

The goal of this paper is to study the number of medium amplitude limit cycles bifurcating from the linear center $\dot{x}=y, \dot{y}=-x$ of the more generalized polynomial Liénard differential systems

$$
\dot{x}=y, \quad \dot{y}=-x-p(x) y-q(x) y^{2} .
$$

More precisely, we consider the planar polynomial differential systems

$$
\dot{x}=y, \quad \dot{y}=-x-\varepsilon\left(p_{1}(x) y+q_{1}(x) y^{2}\right)-\varepsilon^{2}\left(p_{2}(x) y+q_{2}(x) y^{2}\right),
$$

where $p_{j}(x)=\sum_{i=0}^{n} a_{i j} x^{i}$, and $q_{j}(x)=\sum_{i=0}^{n} b_{i j} x^{i}$, for $j=1,2$. Note that such polynomial differential systems have degree $n+2$, because the right hand parts of them are polynomials of degree $n+2$.

A summary of the averaging theory of first and second order for computing limit cycles is given in section 2. Our two main results are the following ones.

Theorem 1. Using the averaging theory of first order the polynomial differential system (2) of degree $n+2$ has at most $\left[\frac{n}{2}\right]$ limit cycles bifurcating from the periodic solutions of the linear center $\dot{x}=y, \dot{y}=-x$. Moreover, this upper bound is reached.

Theorem 1 is proved in section 3 . 
Theorem 2. Using the averaging theory of second order the polynomial differential system (2) of degree $n+2$ has at most $n$ limit cycles bifurcating from the periodic solutions of the linear center $\dot{x}=y, \dot{y}=-x$.

Theorem 2 is proved in section 4 .

Note that for the moment we do not know if the upper bound $n$ for the number of limit cycles of the polynomial differential system (2) of degree $n+2$, which bifurcate from the periodic solutions of the linear center $\dot{x}=y, \dot{y}=-x$, using the averaging method of second order is reached. We conjecture that it is reached, see Remark 13 where the conjecture is proved for $n=1,2,3,4$.

\section{The AVERAgING THEORY OF FIRST AND SECOND ORDER}

The averaging theory for studying specifically limit cycles up to second order in $\varepsilon$ was developed in $[3,21]$. We summarized it here up to second order. The theory up to first order is very classical and can be found for instance in [35].

Consider the differential system

$$
\dot{x}=\varepsilon F_{1}(t, x)+\varepsilon^{2} F_{2}(t, x)+\varepsilon^{3} R(t, x, \varepsilon),
$$

where $F_{1}, F_{2}: \mathbb{R} \times D \rightarrow \mathbb{R}, R: \mathbb{R} \times D \times\left(-\varepsilon_{f}, \varepsilon_{f}\right) \rightarrow \mathbb{R}$ are continuous functions, $T$-periodic in the first variable, and $D$ is an open subset of $\mathbb{R}$. Assume that the following conditions hold.

(i) $F_{1}(t, \cdot) \subset C^{2}(D), F_{2}(t, \cdot) \subset C^{1}(D)$ for all $t \in \mathbb{R}, F_{1}, F_{2}, R$ are locally Lipschitz with respect to $x$, and $R$ is twice differentiable with respect to $\varepsilon$.

We define $F_{k 0}: D \rightarrow \mathbb{R}$ for $k=1,2$ as

$$
\begin{aligned}
& F_{10}(z)=\frac{1}{T} \int_{0}^{T} F_{1}(s, z) d s \\
& F_{20}(z)=\frac{1}{T} \int_{0}^{T}\left[D_{z} F_{1}(s, z)\left(\int_{0}^{s} F_{1}(t, z) d t\right)+F_{2}(s, z)\right] d s
\end{aligned}
$$

(ii) For $V \subset D$ an open and bounded set and for each $\varepsilon \in\left(-\varepsilon_{f}, \varepsilon_{f}\right) \backslash\{0\}$, there exists $a \in V$ such that $F_{10}(a)+\varepsilon F_{20}(a)=0$ and $d_{B}\left(F_{10}+\varepsilon F_{20}, V, a\right) \neq 0$.

Then for $|\varepsilon|>0$ sufficiently small there exists a $T$-periodic solution $x(\cdot, \varepsilon)$ of the system (3) such that $x(0, \varepsilon) \rightarrow a$ when $\varepsilon \rightarrow 0$.

The expression $d_{B}\left(F_{10}+\varepsilon F_{20}, V, a\right) \neq 0$ means that the Brouwer degree of the function $F_{10}+\varepsilon F_{20}: V \rightarrow \mathbb{R}$ at the fixed point $a$ is not zero. A sufficient condition in order that this inequality is true is that the Jacobian of the function $F_{10}+\varepsilon F_{20}$ at $a$ be non-zero.

If $F_{10}$ is not identically zero, then the zeros of $F_{10}+\varepsilon F_{20}$ are mainly the zeros of $F_{10}$ for $\varepsilon$ sufficiently small. In this case the previous result provides the averaging theory of first order.

If $F_{10}$ is identically zero and $F_{20}$ is not identically zero, then the zeros of $F_{10}+\varepsilon F_{20}$ are the zeros of $F_{20}$. In this case the previous result provides the averaging theory of second order. 


\section{Proof of Theorem 1}

If we do in (2) the change of variables $x=r \cos \theta, y=r \sin \theta$ (that is, we use the polar coordinates) we can write system (2) as

$$
\begin{aligned}
& \dot{r}=-r \sin ^{2} \theta\left(\varepsilon\left(p_{1}+q_{1} r \sin \theta\right)+\varepsilon^{2}\left(p_{2}+q_{2} r \sin \theta\right)\right), \\
& \dot{\theta}=-1-\cos \theta \sin \theta\left(\varepsilon\left(p_{1}+q_{1} r \sin \theta\right)+\varepsilon^{2}\left(p_{2}+q_{2} r \sin \theta\right)\right),
\end{aligned}
$$

where $p_{j}=p_{j}(r \cos \theta)$ and $q_{j}=q_{j}(r \cos \theta)$. Therefore we have that

$$
\frac{d r}{d \theta}=\frac{\dot{r}}{\dot{\theta}}=G(\theta, r, \varepsilon)=\varepsilon F_{1}(\theta, r)+\varepsilon^{2} F_{2}(\theta, r)+\ldots,
$$

where $F_{1}(\theta, r)=\left[\frac{\partial}{\partial \varepsilon} G(\theta, r, \varepsilon)\right]_{\varepsilon=0}$ and $F_{2}(\theta, r)=\left[\frac{1}{2} \frac{\partial^{2}}{\partial \varepsilon^{2}} G(\theta, r, \varepsilon)\right]_{\varepsilon=0}$. More precisely, we have

$$
F_{1}(\theta, r)=r \sin ^{2} \theta\left(p_{1}+q_{1} r \sin \theta\right)
$$

and

$$
F_{2}(\theta, r)=r \sin ^{2} \theta\left(p_{2}+q_{2} r \sin \theta\right)-r \sin ^{3} \theta \cos \theta\left(p_{1}+q_{1} r \sin \theta\right)^{2} .
$$

Substituting $p_{1}=\sum_{i=0}^{n} a_{i 1} r^{i} \cos ^{i} \theta$ and $q_{1}=\sum_{i=0}^{n} b_{i 1} r^{i} \cos ^{i} \theta$ in $F_{1}(\theta, r)$ we obtain that

$$
F_{1}(\theta, r)=\sum_{i=0}^{n+1} A_{i}(\theta) r^{i+1}
$$

where

$$
\begin{aligned}
& A_{k}(\theta)=a_{k 1} T_{k}(\theta)+b_{k-1,1} S_{k-1}(\theta), \\
& T_{j}(\theta)=\cos ^{j} \theta-\cos ^{j+2} \theta, \\
& S_{j}(\theta)=\sin \theta \cos ^{j} \theta-\sin \theta \cos ^{j+2} \theta .
\end{aligned}
$$

By convention in the expression of $A_{k}(\theta)$ we have taken

$$
a_{i 1}=0 \text { if } i>n \text {, and } b_{i 1}=0 \text { if } i<0 .
$$

Doing an analogous process with $F_{2}(\theta, r)$ we obtain that

$$
F_{2}(\theta, r)=\sum_{i=0}^{n+1} \tilde{A}_{i}(\theta) r^{i+1}-\left(\sum_{i=0}^{n+1} D_{i}(\theta) r^{i}\right)\left(\sum_{i=0}^{n+1} A_{i}(\theta) r^{i+1}\right),
$$

where

$$
\begin{aligned}
& \tilde{A}_{k}(\theta)=a_{k 2} T_{k}(\theta)+b_{k-1,2} S_{k-1}(\theta), \\
& D_{k}(\theta)=a_{k 1} R_{k+1}(\theta)+b_{k-1,1} T_{k}(\theta), \\
& R_{j}(\theta)=\sin \theta \cos ^{j} \theta .
\end{aligned}
$$

In short, we have reduced the study of the limit cycles of the polynomial system (2) to study the limit cycles of the differential equation (4).

First we shall study the limit cycles of the differential equation (4) using the averaging theory of first order. Therefore, by section 2 we must study the simple positive zeros of the function

$$
F_{10}(r)=\frac{1}{2 \pi} \int_{0}^{2 \pi} F_{1}(\theta, r) d \theta .
$$


For every one of these zeros we will have a limit cycle of the polynomial differential system (2).

If $F_{10}(r)$ is identically zero, applying the theory of averaging of second order (see again section 2 ) every simple positive zero of the function

$$
F_{20}(r)=\frac{1}{2 \pi} \int_{0}^{2 \pi}\left[\frac{\partial F_{1}(\theta, r)}{\partial r}\left(\int_{0}^{\theta} F_{1}(t, r) d t\right)+F_{2}(\theta, r)\right] d \theta
$$

will provide a limit cycle of the polynomial differential system (2).

Taking into account the expression of (5) and (7), in order to obtain $F_{10}(r)$ and $F_{20}(r)$ it is necessary to evaluate the integrals of the form

$$
\int_{0}^{2 \pi} T_{k}(\theta) d \theta \quad \text { and } \quad \int_{0}^{2 \pi} S_{k}(\theta) d \theta
$$

In the following lemmas we compute these integrals.

Lemma 3. Let $\bar{I}_{k}(\theta)=\int_{0}^{\theta} T_{k}(t) d t$. Then

$$
\bar{I}_{k}(\theta)=\sum_{i=0}^{\left[\frac{k+1}{2}\right]} M_{i}(k) \sin \theta \cos ^{k+1-2 i} \theta+\left(k+1-2\left[\frac{k+1}{2}\right]\right) M_{\left[\frac{k+1}{2}\right]}(k) \theta,
$$

where

$$
\begin{aligned}
& M_{0}(k)=-\frac{1}{k+2} \\
& M_{1}(k)=\frac{1}{k(k+2)} \\
& M_{j}(k)=M_{j-1}(k) \frac{k-2 j+3}{k-2 j+2} \quad \text { for each } j=2,3, \ldots,\left[\frac{k+1}{2}\right] .
\end{aligned}
$$

Moreover

$$
\bar{I}_{k}(2 \pi)=\left\{\begin{array}{cl}
0 & \text { if } \quad k \text { is odd }, \\
\left(\begin{array}{c}
k \\
k / 2
\end{array}\right) \frac{\pi}{(k+2) 2^{k-1}} & \text { if } k \text { is even. }
\end{array}\right.
$$

Proof. In order to prove the value of $\bar{I}_{k}(\theta)$ given in (10), it is sufficient to prove that its derivative is $T_{k}(\theta)$. Now, if we derive the expression of the second right hand part of (10) with respect to $\theta$, we obtain

$$
\begin{aligned}
& \sum_{i=0}^{\left[\frac{k+1}{2}\right]} M_{i}(k)\left(\cos ^{k+2-2 i} \theta-(k+1-2 i)\left(1-\cos ^{2} \theta\right) \cos ^{k-2 i} \theta\right) \\
& +\left(k+1-2\left[\frac{k+1}{2}\right]\right) M_{\left[\frac{k+1}{2}\right]}(k),
\end{aligned}
$$

that is,

$$
\begin{aligned}
& \sum_{i=0}^{\left[\frac{k+1}{2}\right]} M_{i}(k)\left((k+2-2 i) \cos ^{k+2-2 i} \theta-(k+1-2 i) \cos ^{k-2 i} \theta\right) \\
& +\left(k+1-2\left[\frac{k+1}{2}\right]\right) M_{\left[\frac{k+1}{2}\right]}(k),
\end{aligned}
$$


that can be written in the form

$$
\begin{aligned}
& (k+2) M_{0}(k) \cos ^{k+2} \theta+\sum_{i=1}^{\left[\frac{k+1}{2}\right]} M_{i}(k)(k+2-2 i) \cos ^{k+2-2 i} \theta \\
& -\sum_{i=0}^{\left[\frac{k+1}{2}\right]-1} M_{i}(k)(k+1-2 i) \cos ^{k-2 i} \theta-M_{\left[\frac{k+1}{2}\right]}(k)\left(k+1-2\left[\frac{k+1}{2}\right]\right) \cos ^{k-2[(k+1) / 2]} \theta \\
& +\left(k+1-2\left[\frac{k+1}{2}\right]\right) M_{\left[\frac{k+1}{2}\right]}(k) .
\end{aligned}
$$

Taking into account that

$$
\sum_{i=0}^{\left[\frac{k+1}{2}\right]-1} M_{i}(k)(k+1-2 i) \cos ^{k-2 i} \theta=\sum_{j=1}^{\left[\frac{k+1}{2}\right]} M_{j-1}(k)(k+3-2 j) \cos ^{k+2-2 j} \theta,
$$

the expression of $\frac{d \bar{I}_{k}(\theta)}{d \theta}$ becomes

$$
\sum_{i=0}^{\left[\frac{k+1}{2}\right]} N_{i}(k) \cos ^{k+2-2 i} \theta+\left(k+1-2\left[\frac{k+1}{2}\right]\right) M_{\left[\frac{k+1}{2}\right]}(k)\left(1-\cos ^{k-2\left[\frac{k+1}{2}\right]} \theta\right),
$$

where

$$
\begin{aligned}
& N_{0}(k)=(k+2) M_{0}(k), \\
& N_{i}(k)=\left((k-2 i+2) M_{i}(k)-(k-2 i+3) M_{i-1}(k)\right), \quad \text { for } i \geq 2 .
\end{aligned}
$$

Using (11) we deduce that $N_{0}(k)=-1, N_{1}(k)=1$ and $N_{i}(k)=0$ for $i \geq 2$. Finally we note that if $k$ is odd then $k+1-2\left[\frac{k+1}{2}\right]=0$, and if $k$ is even then $1-\cos ^{k-2\left[\frac{k+1}{2}\right]} \theta=0$. Hence the value of (13) is $\cos ^{k} \theta-\cos ^{k+2} \theta$, that is $T_{k}(\theta)$. Therefore (10) is proved.

Now we shall compute the value of $\bar{I}_{k}(2 \pi)$. First we observe that $k+1-2\left[\frac{k+1}{2}\right]$ is 0 if $k$ is odd. Therefore, from (10) we have that

$$
\bar{I}_{k}(2 \pi)=\sum_{i=0}^{\left[\frac{k+1}{2}\right]} M_{i}(k) \sin (2 \pi) \cos ^{k+1-2 i}(2 \pi)=0 .
$$

On the other hand, when $k$ is even $k+1-2\left[\frac{k+1}{2}\right]=1$ and, taking into account (11) we have

$$
M_{\left[\frac{k+1}{2}\right]}(k)=M_{\frac{k}{2}}(k)=\frac{(k-1)(k-3) \ldots 3}{(k+2) k(k-2) \ldots 2}=\left(\begin{array}{c}
k \\
k / 2
\end{array}\right) \frac{1}{(k+2) 2^{k}},
$$

and hence, again from (10) we obtain

$$
\bar{I}_{k}(2 \pi)=\sum_{i=0}^{\left[\frac{k+1}{2}\right]} M_{i}(k) \sin (2 \pi) \cos ^{k+1-2 i}(2 \pi)+\left(\begin{array}{c}
k \\
k / 2
\end{array}\right) \frac{\pi}{(k+2) 2^{k-1}} .
$$

This completes the proof of the lemma. 
Lemma 4. Let $J_{k}(\theta)=\int_{0}^{\theta} \sin t \cos ^{k} t d t$ and $\bar{J}_{k}(\theta)=\int_{0}^{\theta} S_{k}(t) d t=J_{k-2}(\theta)-J_{k}(\theta)$. Then the following equalities hold:

$$
\begin{aligned}
& \bar{J}_{k}(\theta)=\frac{2}{(k+1)(k+3)}+\frac{1}{k+3} \cos ^{k+3} \theta-\frac{1}{k+1} \cos ^{k+1} \theta, \\
& \bar{J}_{k}(2 \pi)=0
\end{aligned}
$$

for each value de $k$.

Proof. Both equalities follow easily by direct computation.

Using these two lemmas we shall obtain in the next proposition the integral the function $F_{10}(r)$. First, we define the function

$$
\mathbb{I}_{\theta}(r)=\int_{0}^{\theta} F_{1}(t, r) d t
$$

Then taking into account the expression of (5), we deduce that

$$
\mathbb{I}_{\theta}(r)=\sum_{i=0}^{n+1} B_{i}(\theta) r^{i+1}
$$

where $B_{i}(\theta)=\int_{0}^{\theta} A_{i}(\theta) d \theta$, that is

$$
B_{i}(\theta)=a_{i 1} \bar{I}_{k}(\theta)+b_{i-1,1} \bar{J}_{i-1}(\theta) .
$$

Proposition 5. We have

$$
F_{10}(r)=\frac{\mathbb{I}_{2 \pi}(r)}{2 \pi}=r\left(\sum_{i=0}^{[n / 2]} \frac{a_{2 i, 1}}{2^{2 i+1}(i+1)}\left(\begin{array}{c}
2 i \\
i
\end{array}\right) r^{2 i}\right) .
$$

Proof. Since $\mathbb{I}_{2 \pi}(r)$ can be obtained using (15) with $\theta=2 \pi$, we must determine the values of $B_{i}(2 \pi)$. Using (16) and, taking into account that $\bar{J}_{i-1}(2 \pi)=0$ (see Lemma 4 ), we have that $B_{i}(2 \pi)=a_{i 1} \bar{I}_{k}(2 \pi)$. Now, if we calcule $B_{i}(2 \pi)$, by using the expression for $\bar{I}_{k}(2 \pi)$ obtained in (12), it follows (17).

Proof of Theorem 1. From Proposition 5 the function $F_{10}(r)$ has at most $\left[\frac{n}{2}\right]$ simple positive zeros. Furthermore, since we can choose arbitrary values for $a_{2 i, 1}$ and, in addition, these coefficients appear multiplied by nonzero constants, it is possible to reach this upper bound. Therefore, using the averaging theory of first order, the polynomial differential system (2) has at most $\left[\frac{n}{2}\right]$ limit cycles bifurcating from the periodic solutions of the linear center $\dot{x}=y, \dot{y}=-x$ and, by choosing an adequate system, one can has exactly these number of limit cycles.

\section{Proof of Theorem 2}

When $\mathbb{I}_{2 \pi}(r) \equiv 0$ we shall apply the second order averaging theory for studying the limit cycles of the polynomial differential system (2). Therefore, from (9) we must study the simple positive zeros of the function

$$
F_{20}(r)=\frac{1}{2 \pi} \int_{0}^{2 \pi}\left(\frac{\partial F_{1}(\theta, r)}{\partial r} \mathbb{I}_{\theta}(r)+F_{2}(\theta, r)\right) d \theta .
$$


We split the computation of the function $F_{20}(r)$ in two pieces, i.e. we define $2 \pi F_{20}(r)=\mathbb{L}(r)+\mathbb{J}(r)$, where

$$
\mathbb{L}(r)=\int_{0}^{2 \pi} \frac{\partial F_{1}(\theta, r)}{\partial r} \mathbb{I}_{\theta}(r) d \theta \quad \text { and } \quad \mathbb{J}(r)=\int_{0}^{2 \pi} F_{2}(\theta, r) d \theta .
$$

First we shall calcule the function $\mathbb{L}(r)$.

From (5) and (15) we get

$$
\begin{aligned}
\mathbb{L}(r)= & \sum_{k=1}^{n+2}\left(\sum_{j=0}^{k-1}(j+1) \int_{0}^{2 \pi} A_{j}(\theta) B_{k-j-1}(\theta) d \theta\right) r^{k}+ \\
& \sum_{k=1}^{n+1}\left(\sum_{j=k}^{n+1}(j+1) \int_{0}^{2 \pi} A_{j}(\theta) B_{n+k-j+1}(\theta) d \theta\right) r^{n+k+2} .
\end{aligned}
$$

Remark 6. We have that $\int_{0}^{2 \pi} A_{k}(\theta) B_{k}(\theta) d \theta=0$. Indeed, we have that $A_{k}(\theta)$ is the derivative of $B_{k}(\theta)$. Hence a primitive function of the integrand is $B_{k}(\theta)^{2} / 2$ and taking into account (16) and Lemmas 3 and 4 , we deduce that the values of the primitive function at $2 \pi$ and at 0 coincide.

Now we shall compute the integrals $\int_{0}^{2 \pi} A_{p}(\theta) B_{q}(\theta) d \theta$ that appear in (18) when $p \neq q$. Using the expression of $A_{k}(\theta)$ and (16) we have

$$
\begin{aligned}
\int_{0}^{2 \pi} A_{p}(\theta) B_{q}(\theta) d \theta= & a_{p 1} a_{q 1} \int_{0}^{2 \pi} T_{p}(\theta) \bar{I}_{q}(\theta) d \theta+ \\
& a_{p 1} b_{q-1,1} \int_{0}^{2 \pi} T_{p}(\theta) \bar{J}_{q-1}(\theta) d \theta+ \\
& b_{p-1,1} a_{q 1} \int_{0}^{2 \pi} S_{p-1}(\theta) \bar{I}_{q}(\theta) d \theta+ \\
& b_{p-1,1} b_{q-1,1} \int_{0}^{2 \pi} S_{p-1}(\theta) \bar{J}_{q-1}(\theta) d \theta .
\end{aligned}
$$

In the next proposition we obtain some results on the integrals of the right hand part of (19).

Proposition 7. If $p \neq q$ the following statements hold.

(a) $\int_{0}^{2 \pi} S_{p-1}(\theta) \bar{J}_{q-1}(\theta) d \theta=0$ for all values of $p$ and $q$.

(b) If $q$ is odd, then $\int_{0}^{2 \pi} T_{p}(\theta) \bar{I}_{q}(\theta) d \theta=0$.

(c) If $p$ is odd, then $\int_{0}^{2 \pi} T_{p}(\theta) \bar{J}_{q-1}(\theta) d \theta$ takes the value 0 if $q$ is even, and the value

$$
-\frac{\pi(2 p+5 q+8)}{2^{p+q-1} q(q+2)(p+q+2)(p+q+4)}\left(\begin{array}{c}
p+q \\
(p+q) / 2
\end{array}\right)
$$

if $q$ is odd. 
(d) If $q$ is odd, then $\int_{0}^{2 \pi} \bar{I}_{q}(\theta) S_{p-1}(\theta) d \theta$ takes the value 0 if $p$ is even, and the value

$$
\sum_{i=0}^{(q+1) / 2} \frac{3 \pi M_{i}(q)}{2^{p+q-1-2 i}(p+q+2-2 i)(p+q+4-2 i)}\left(\begin{array}{c}
p+q-2 i \\
(p+q-2 i) / 2
\end{array}\right)
$$

if $p$ is odd, where $M_{i}(q)$ is defined in Lemma 3.

Proof. From the definition of the function $S_{j}(\theta)$ and (14) we obtain that the $2 \pi-$ periodic function $S_{p-1}(\theta) \bar{J}_{q-1}(\theta)$ is odd. Therefore statement (a) follows.

If $q$ is odd, then

$$
T_{p}(\theta) \bar{I}_{q}(\theta)=\left(\cos ^{p} \theta-\cos ^{p+2} \theta\right)\left(\sum_{i=0}^{\frac{q+1}{2}} M_{i}(q) \sin \theta \cos ^{q+1-2 i} \theta\right) .
$$

This function again is odd and $2 \pi$-periodic, therefore statement (b) is proved.

Since

$$
T_{p}(\theta) \bar{J}_{q-1}(\theta)=\left(\cos ^{p} \theta-\cos ^{p+2} \theta\right)\left(\frac{2}{q(q+2)}+\frac{1}{q+2} \cos ^{q+2} \theta-\frac{1}{q} \cos ^{q} \theta\right),
$$

from the definition of $T_{p}$, we obtain that

$$
T_{p}(\theta) \bar{J}_{q-1}(\theta)=\frac{2}{q(q+2)} T_{p}(\theta)+\frac{1}{q+2} T_{p+q+2}(\theta)-\frac{1}{q} T_{p+q}(\theta) .
$$

Hence, taking into account the definition of $\bar{I}_{k}(\theta)$, we deduce that

$$
\int_{0}^{2 \pi} T_{p}(\theta) \bar{J}_{q-1}(\theta) d \theta=\frac{2}{q(q+2)} \bar{I}_{p}(2 \pi)+\frac{1}{q+2} \bar{I}_{p+q+2}(2 \pi)-\frac{1}{q} \bar{I}_{p+q}(2 \pi) .
$$

Now if we take into account the values of $\bar{I}_{k}(2 \pi)$ given in Lemma 3 , statement (c) follows.

Finally taking into account that

$$
\begin{aligned}
\bar{I}_{q}(\theta) S_{p-1}(\theta) & =\left(\sum_{i=0}^{\frac{q+1}{2}} M_{i}(q) \sin \theta \cos ^{q+1-2 i} \theta\right)\left(\sin \theta \cos ^{p-1} \theta-\sin \theta \cos ^{p+1} \theta\right) \\
& =\left(1-\cos ^{2} \theta\right)\left(\sum_{i=0}^{\frac{q+1}{2}} M_{i}(q) \cos ^{q+1-2 i} \theta\right)\left(\cos ^{p-1} \theta-\cos ^{p+1} \theta\right),
\end{aligned}
$$

again we can express $\bar{I}_{q}(\theta) S_{p-1}(\theta)$ in terms of the integrals of the functions $T_{i}$, and using again the values of $\bar{I}_{k}(2 \pi)$ given in Lemma 3 , statement (d) follows.

Proposition 8. If $\mathbb{I}_{2 \pi}(r) \equiv 0$, then

$$
\begin{aligned}
\mathbb{L}(r)= & \sum_{k=1}^{\left[\frac{n}{2}\right]}\left(\sum_{j=0}^{k-1}(2 j+2) L(k, j)\right) r^{2 k+1}+ \\
& \sum_{k=0}^{\left[\frac{n}{2}\right]}\left(\sum_{j=k}^{\left[\frac{n}{2}\right]}(2 j+2) L\left(k+\left[\frac{n+2}{2}\right], j\right)\right) r^{2\left(k+\left[\frac{n+2}{2}\right]\right)+1},
\end{aligned}
$$


where

$$
\begin{aligned}
L(t, j)= & -a_{2 j+1,1} b_{2 t-2 j-2,1} \frac{\pi\left(\begin{array}{c}
2 t \\
t
\end{array}\right)(10 t-6 j+5)}{2^{2 t+1}(t+1)(t+2)(2 t-2 j-1)(2 t-2 j+1)} \\
& +b_{2 j, 1} a_{2 t-2 j-1,1} \sum_{i=0}^{t-j} \frac{3 \pi M_{i}(2 t-2 j-1)}{2^{2 t-2 i+1}(t+1-i)(t+2-i)}\left(\begin{array}{c}
2 t-2 i \\
t-i
\end{array}\right) .
\end{aligned}
$$

Proof. If $\mathbb{I}_{2 \pi}(r)=0$, using Proposition 5, we have that $a_{i 1}=0$ when $i$ is even. Then we shall prove that $\int_{0}^{2 \pi} A_{p}(\theta) B_{q}(\theta) d \theta=0$ if $p$ or $q$ are even. If $p$ is even, from (19) and statement (a) of Proposition 7, we get

$$
\int_{0}^{2 \pi} A_{p}(\theta) B_{q}(\theta) d \theta=b_{p-1,1} a_{q 1} \int_{0}^{2 \pi} S_{p-1}(\theta) \bar{I}_{q}(\theta) d \theta .
$$

Therefore using that $a_{q 1}=0$ if $q$ is even and statement (d) of Proposition 7 if $q$ is odd, it follows that the above integral is 0 .

If $q$ is even, using again statement (a) of Proposition 7, we obtain

$$
\int_{0}^{2 \pi} A_{p}(\theta) B_{q}(\theta) d \theta=a_{p 1} b_{q-1,1} \int_{0}^{2 \pi} T_{p}(\theta) \bar{J}_{q-1}(\theta) d \theta .
$$

In this case using that $a_{p 1}=0$ if $p$ is even, and statement (c) of Proposition 7 if $p$ is odd, again we obtain that the above integral is 0 .

On the other hand in the case that $p$ and $q$ are odd, using statements (a) and (b) of Proposition 7 we can write (19) as

$$
\int_{0}^{2 \pi} A_{p}(\theta) B_{q}(\theta) d \theta=a_{p 1} b_{q-1,1} \int_{0}^{2 \pi} T_{p}(\theta) \bar{J}_{q-1}(\theta) d \theta+b_{p-1,1} a_{q 1} \int_{0}^{2 \pi} S_{p-1}(\theta) \bar{I}_{q}(\theta) d \theta .
$$

The integrals on the right hand part can be calculated using statements (c) and (d) of Proposition 7. If we remove from (18) all the integrals where $p$ or $q$ are even and we substitute the others integrals by their values, we obtain (20) and the proposition follows.

We note that some of the values of $L(t, j)$ are zero. Indeed, the integral that corresponds to $t=(j-1) / 2$ is $\int_{0}^{2 \pi} A_{2 j+1}(\theta) B_{2 j+1}(\theta) d \theta$, and from Remark 6 it is
zero.

In order to complete the computation of $F_{20}(r)$ we must determine the function $\mathbb{J}(r)$. Taking in account the expression of $F_{2}(\theta, r)$ given in (7), first we compute the integral $\int_{0}^{2 \pi} \sum_{i=0}^{n+1} \tilde{A}_{i}(\theta) d \theta$.

Lemma 9. We have

$$
\int_{0}^{2 \pi} \sum_{i=0}^{n+1} \tilde{A}_{i}(\theta) d \theta=\pi \sum_{i=0}^{[n / 2]} \frac{a_{2 i, 2}}{2^{2 i}(i+1)}\left(\begin{array}{c}
2 i \\
i
\end{array}\right) .
$$

Proof. The proof is analogous to the proof of Proposition 5. 
Next we shall compute the function

$$
\mathbb{S}(r)=\int_{0}^{2 \pi}\left(\sum_{i=0}^{n+1} D_{i}(\theta) r^{i}\right)\left(\sum_{i=0}^{n+1} A_{i}(\theta) r^{i+1}\right) d \theta
$$

i.e.

$$
\begin{aligned}
\mathbb{S}(r)= & \sum_{k=1}^{n+2}\left(\sum_{j=0}^{k-1} \int_{0}^{2 \pi} D_{j}(\theta) A_{k-j-1}(\theta) d \theta\right) r^{k}+ \\
& \sum_{k=1}^{n+1}\left(\sum_{j=k}^{n+1} \int_{0}^{2 \pi} D_{j}(\theta) A_{n+k-j+1}(\theta) d \theta\right) r^{n+k+2} .
\end{aligned}
$$

Using the definitions of the functions $A_{q}(\theta)$ and $D_{p}(\theta)$ we have

$$
\begin{aligned}
\int_{0}^{2 \pi} D_{p}(\theta) A_{q}(\theta) d \theta= & a_{p 1} a_{q 1} \int_{0}^{2 \pi} R_{p+1}(\theta) T_{q}(\theta) d \theta+ \\
& a_{p 1} b_{q-1,1} \int_{0}^{2 \pi} R_{p+1}(\theta) S_{q-1}(\theta) d \theta+ \\
& +b_{p-1,1} a_{q 1} \int_{0}^{2 \pi} T_{p}(\theta) T_{q}(\theta) d \theta+ \\
& b_{p-1,1} b_{q-1,1} \int_{0}^{2 \pi} T_{p}(\theta) S_{q-1}(\theta) d \theta
\end{aligned}
$$

In the next proposition we obtain some results on the integrals of the right hand part of (22).

Proposition 10. The following statements hold.

(a) $\int_{0}^{2 \pi} R_{p+1}(\theta) T_{q}(\theta) d \theta=0$

(b) If $p$ is odd, then $\int_{0}^{2 \pi} R_{p+1}(\theta) S_{q-1}(\theta) d \theta$ is zero if $q$ is even, and equal to

$$
\frac{3 \pi\left(\begin{array}{c}
p+q \\
(p+q) / 2
\end{array}\right)}{2^{p+q-1}(p+q+2)(p+q+4)}
$$

if $q$ is odd.

(c) If $q$ is odd, then $\int_{0}^{2 \pi} T_{p}(\theta) T_{q}(\theta) d \theta$ is zero if $p$ is even, and equal to

$$
\frac{3 \pi\left(\begin{array}{c}
p+q \\
(p+q) / 2
\end{array}\right)}{2^{p+q-1}(p+q+2)(p+q+4)},
$$

if $p$ is odd.

(d) $\int_{0}^{2 \pi} T_{p}(\theta) S_{q-1}(\theta) d \theta=0$.

Proof. The proof of this proposition is similar to the proof of Proposition 7. 
Proposition 11. If $\mathbb{I}_{2 \pi}(r) \equiv 0$, then

$$
\begin{aligned}
\mathbb{S}(r)= & \sum_{k=1}^{\left[\frac{n}{2}\right]}\left(\sum_{j=0}^{k-1} S(k, j)\right) r^{2 k+1}+ \\
& \sum_{k=0}^{\left[\frac{n}{2}\right]}\left(\sum_{j=k}^{\left[\frac{n}{2}\right]} S\left(k+\left[\frac{n+2}{2}\right], j\right)\right) r^{2\left(k+\left[\frac{n+2}{2}\right]\right)+1},
\end{aligned}
$$

where

$$
S(t, j)=\frac{3 \pi\left(\begin{array}{c}
2 t \\
t
\end{array}\right)\left(a_{2 j+1,1} b_{2 t-2 j-2,1}+b_{2 j, 1} a_{2 t-2 j-1,1}\right)}{2^{2 t+1}(t+1)(t+2)} .
$$

Proof. Since $\mathbb{I}_{2 \pi}(r) \equiv 0$, by Proposition 5 , the coefficients $a_{i 1}=0$ when $i$ is even. Then we shall prove that $\int_{0}^{2 \pi} D_{p}(\theta) A_{q}(\theta) d \theta=0$ if $p$ or $q$ are even. If $p$ is even, from (22) and statement (d) of Proposition 10, we have

$$
\int_{0}^{2 \pi} D_{p}(\theta) A_{q}(\theta) d \theta=b_{p-1,1} a_{q 1} \int_{0}^{2 \pi} T_{p}(\theta) T_{q}(\theta) d \theta .
$$

Then using that $a_{q 1}=0$ if $q$ is even and statement (c) of Proposition 10 if $q$ is odd, we obtain that the above integral is 0 .

If $q$ is even, using statement (a) of Proposition 10 we get

$$
\int_{0}^{2 \pi} D_{p}(\theta) A_{q}(\theta) d \theta=a_{p 1} b_{q-1,1} \int_{0}^{2 \pi} R_{p+1}(\theta) S_{q-1}(\theta) d \theta .
$$

Then, since $a_{p 1}=0$ if $p$ is even, and from statement (b) of Proposition 10 if $p$ is odd, we obtain that the above integral is 0 .

In the case that $p$ and $q$ are odd, using statements (a) and (b) of Proposition 10 we can write (22) as

$$
\int_{0}^{2 \pi} D_{p}(\theta) A_{q}(\theta) d \theta=a_{p 1} b_{q-1,1} \int_{0}^{2 \pi} R_{p+1}(\theta) S_{q-1}(\theta) d \theta+b_{p-1,1} a_{q 1} \int_{0}^{2 \pi} T_{p}(\theta) T_{q}(\theta) d \theta
$$

that can be calculated using statements (b) and (c) of Proposition 10. If we remove from the definition of the function $\mathbb{S}(r)$ all the integrals where $p$ or $q$ are even, and we substitute the others integrals by their values, we obtain (23), and the proposition is proved.

Proposition 12. If $\mathbb{I}_{2 \pi}(r) \equiv 0$, then the function $F_{20}(r)$ defined in (9) can be expressed as $\frac{r}{2} \tilde{H}(r)$ where

$$
\tilde{H}(r)=H_{0}+\sum_{k=1}^{\left[\frac{n}{2}\right]} H_{k} r^{2 k}+\sum_{k=0}^{\left[\frac{n-1}{2}\right]} H_{k+\left[\frac{n+2}{2}\right]} r^{2\left(k+\left[\frac{n+2}{2}\right]\right)},
$$


with

$$
\begin{aligned}
H_{0}= & a_{02}, \\
H_{k}= & \frac{a_{2 k, 2}\left(\begin{array}{c}
2 k \\
k
\end{array}\right)}{2^{2 k}(k+1)}+\sum_{j=0}^{k-1} a_{2 j+1,1} b_{2 k-2 j-2,1}(C(k, j)+E(k, k-j-1)), \\
H_{k+\left[\frac{n+2}{2}\right]=} & \sum_{j=k}^{\left[\frac{n-1}{2}\right]} a_{2 j+1,1} b_{2\left(k+\left[\frac{n+2}{2}\right]\right)-2 j-2,1}\left(C\left(k+\left[\frac{n+2}{2}\right], j\right)+\right. \\
& \left.E\left(k+\left[\frac{n+2}{2}\right], k+\left[\frac{n+2}{2}\right]-j-1\right)\right)
\end{aligned}
$$

and

$$
\begin{aligned}
C(t, j) & =-\left(\frac{(2 j+2)(10 t-6 j+5)}{(2 t-2 j-1)(2 t-2 j+1)}+3\right) \frac{\left(\begin{array}{c}
2 t \\
t
\end{array}\right)}{2^{2 t+1}(t+1)(t+2)}, \\
E(t, j) & =\sum_{i=0}^{t-j}(2 j+2) \frac{3 M_{i}(2 t-2 j-1)\left(\begin{array}{c}
2 t-2 i \\
t-i
\end{array}\right)}{2^{2 t-2 i+1}(t+1-i)(t+2-i)}-\frac{3\left(\begin{array}{c}
2 t \\
t
\end{array}\right)}{2^{2 t+1}(t+1)(t+2)} .
\end{aligned}
$$

Proof. Since $2 \pi F_{20}(r)=\mathbb{L}(r)+\mathbb{J}(r)$ and

$$
\mathbb{J}(r)=\left(\int_{0}^{2 \pi} \sum_{i=0}^{n+1} \tilde{A}_{i}(\theta) d \theta\right) r^{i+1}-\mathbb{S}(r),
$$

using Propositions 8 and 11 and Lemma 9, we have

$$
\begin{aligned}
2 \pi F_{20}(r)= & \pi\left(\sum_{i=0}^{[n / 2]} \frac{a_{2 i, 2}}{2^{2 i}(i+1)}\left(\begin{array}{c}
2 i \\
i
\end{array}\right) r^{2 i+1}\right)+ \\
& \sum_{k=1}^{\left[\frac{n}{2}\right]}\left(\sum_{j=0}^{k-1}(2 j+2) L(k, j)-S(k, j)\right) r^{2 k+1}+ \\
& \sum_{k=0}^{\left[\frac{n}{2}\right]}\left(\sum_{j=k}^{\left[\frac{n}{2}\right]}(2 j+2) L\left(k+\left[\frac{n+2}{2}\right], j\right)-S\left(k+\left[\frac{n+2}{2}\right], j\right)\right) r^{2\left(k+\left[\frac{n+2}{2}\right]\right)+1} .
\end{aligned}
$$

From (21) and (24) we get

$$
(2 j+2) L(t, j)-S(t, j)=\pi\left(a_{2 j+1,1} b_{2 t-2 j-2,1} C(t, j)+b_{2 j, 1} a_{2 t-2 j-1,1} E(t, j)\right),
$$

and consequently

$$
\tilde{H}(r)=H_{0}+\sum_{k=1}^{\left[\frac{n}{2}\right]} H_{k} r^{2 k}+\sum_{k=0}^{\left[\frac{n}{2}\right]} H_{k+\left[\frac{n+2}{2}\right]} r^{2\left(k+\left[\frac{n+2}{2}\right]\right)}
$$


where

$$
\begin{aligned}
H_{0} & =a_{02} \\
H_{k} & =\frac{a_{2 k, 2}\left(\begin{array}{c}
2 k \\
k
\end{array}\right)}{2^{2 k}(k+1)}+\sum_{j=0}^{k-1}\left(a_{2 j+1,1} b_{2 k-2 j-2,1} C(k, j)+b_{2 j, 1} a_{2 k-2 j-1,1} E(k, j)\right) \\
H_{k+\left[\frac{n+2}{2}\right]}= & \sum_{j=k}^{\left[\frac{n}{2}\right]}\left(a_{2 j+1,1} b_{2\left(k+\left[\frac{n+2}{2}\right]\right)-2 j-2,1} C\left(k+\left[\frac{n+2}{2}\right], j\right)+\right. \\
& \left.b_{2 j, 1} a_{2\left(k+\left[\frac{n+2}{2}\right]\right)-2 j-1,1} E\left(k+\left[\frac{n+2}{2}\right], j\right)\right) .
\end{aligned}
$$

Now we observe that if $t$ is fixed and $j_{2}=t-j_{1}-1$, then $a_{2 j_{1}+1,1} b_{2 t-2 j_{1}-2,1}=$ $b_{2 j_{2}, 1} a_{2 t-2 j_{2}-1,1}$. Using this relation we can write $H_{k}$ and $H_{k+\left[\frac{n+2}{2}\right]}$ as they appear in the statement of the proposition. We note that now the sum in $H_{k+\left[\frac{n+2}{2}\right]}$ ends with $j=[(n-1) / 2]$, because if $n$ is even the term that corresponds to $j=\left[\frac{n}{2}\right]$ is zero, see (6). This completes the proof of the proposition.

Proof of Theorem 2. From (25), the coefficient of the highest degree of $\tilde{H}(r)$ has exponent $2\left(\left[\frac{n-1}{2}\right]+\left[\frac{n+2}{2}\right]\right)$. Since $\left[\frac{n-1}{2}\right]+\left[\frac{n+2}{2}\right]$ takes the value $(n-1) / 2+(n+1) / 2$ if $n$ is odd, and the value $(n-2) / 2+(n+2) / 2$ if $n$ is even, that is $n$ in both cases, then the polynomial $\tilde{H}(r)$ has degree at most $2 n$.

Taking into account the above arguments, we deduce that $F_{20}(r)=\frac{r}{2} \tilde{H}(r)$ can has at most $n$ simple positive zeros, and consequently the polynomial differential system (2) will have at most $n$ limit cycles bifurcating from the periodic solutions of the linear center $\dot{x}=y, \dot{y}=-x$, using the averaging theory of second order.

Remark 13. In Section 1 we conjecture that the upper bound $n$ of Theorem 2 can be reached and, in fact, our numerical computations confirm this idea. For example, in the following we prove that the bound is reached for the values of $n=1,2,3,4$.

If we denote by $\tilde{F}_{n}(r)$ the function $\tilde{H}(r)=2 F_{20}(r) / r$ corresponding to degree $n$, an adequate computation allows to obtain that

$$
\begin{gathered}
\tilde{F}_{1}(r)=a_{02}-(1 / 4) a_{11} b_{01} r^{2}, \\
\tilde{F}_{2}(r)=\tilde{F}_{1}(r)+(1 / 4) r^{2}\left(a_{22}-(1 / 6) a_{11} b_{21} r^{2}\right), \\
\tilde{F}_{3}(r)=\tilde{F}_{2}(r)-(1 / 8) r^{4} a_{31}\left((7 / 3) b_{01}+(3 / 8) b_{21} r^{2}\right),
\end{gathered}
$$

and

$$
\tilde{F}_{4}(r)=\tilde{F}_{3}(r)+(1 / 8) r^{4}\left(a_{42}-(1 / 8) a_{11} b_{41} r^{2}-(11 / 80) a_{31} b_{41} r^{4}\right) .
$$

Now if we fix $a_{02}=1, a_{11}=2$ and $b_{01}=2$ we have that $\tilde{F}_{1}(r)=1-r^{2}$, that has exactly one positive zero. If we fix again $a_{02}=1, a_{11}=2$ and $b_{01}=2$ and furthermore we choose $a_{22}=-1$ and $b_{21}=-3$, we have $\tilde{F}_{2}(r)=\frac{1}{4}\left(1-r^{2}\right)\left(4-r^{2}\right)$ that has exactly two positive zeros.

On the other hand, if we choose $a_{02}=1, a_{11}=0, b_{01}=-\frac{4}{3}, a_{22}=-\frac{49}{9}$, $b_{21}=\frac{16}{27}$, and $a_{31}=1$, then $\tilde{F}_{3}(r)=\frac{1}{36}\left(1-r^{2}\right)\left(4-r^{2}\right)\left(9-r^{2}\right)$ has exactly three positive zeros. Finally if we choose $a_{02}=1, a_{11}=0, b_{01}=0, a_{22}=-\frac{205}{36}, b_{21}=1$, $a_{31}=\frac{10}{9}, a_{42}=\frac{91}{24}$ and $b_{41}=-\frac{1}{11}$, then $\tilde{F}_{4}(r)=\frac{1}{576}\left(1-r^{2}\right)\left(4-r^{2}\right)\left(9-r^{2}\right)\left(16-r^{2}\right)$ has exactly four positive zeros. 


\section{ACKNOWLEDGMENTS}

The first and the third authors are partially supported by a MTM2011-22956. The second author is supported by a MINECO/FEDER grant number MTM200803437, by an AGAUR grant number 2009SGR 410, by ICREA Academia and by FP7-PEOPLE-2012-IRSES 316338 and 318999.

\section{REFERENCES}

[1] J. Alavez-Ramirez, G. Blé, J. Llibre and J. Lopez-Lopez, On the maximum number of limit cycles of a class of generalized Liénard differential systems, Int. J. Bifurcation and Chaos 22 (2012), 1250063-14 pp.

[2] T.R. Blows AND N. G. Lloyd, The number of small-amplitude limit cycles of Liénard equations, Math. Proc. Camb. Phil. Soc. 95 (1984), 359-366.

[3] A. Buic̆ And J. Llibre, Averaging methods for finding periodic orbits via Brouwer degree, Bull. Sci. Math. 128 (2004), 7-22.

[4] X. Chen, J. Llibre and Z. Zhang, Sufficient conditions for the existence of at least $n$ or exactly $n$ limit cycles for the Lienard differential systems, J. Differential Equations 242 (2007), 11-23.

[5] C.J. Christopher And S. Lynch, Small-amplitude limti cycle bifurcations for Liénard systems with quadratic or cubic dapimg or restoring forces, Nonlinearity 12 (1999), 1099-1112.

[6] W. A. Coppel, Some quadratic systems with at most one limit cycles, Dynamics Reported, Vol.2, Wiley, New York, 1998, pp. 61-68.

[7] P. De Maesschalck and F. Dumortier, Classical Liénard equations of degree $n \geq 6$ can have $[(n-1) / 2]+2$ limit cycles, J. Differential Equations 250 (2011), 2162-2176.

[8] F. Dumortier, D. Panazzolo and R. Roussarie, More limit cycles than expected in Liénard systems, Proc. Amer. Math. Soc. 135 (2007), 1895-1904.

[9] F. Dumortier And C. LI, On the uniqueness of limit cycles surrounding one or more singularities for Liénard equations, Nonlinearity 9 (1996), 1489-1500.

[10] F. Dumortier AND C. LI, Quadratic Liénard equations with quadratic damping, J. Differential Equations. 139 (1997), 41-59.

[11] F. Dumortier and C. Rousseau, Cubic Liénard equations with linear dapimg, Nonlinearity 3 (1990), 1015-1039.

[12] A. Gasull and J. Torregrosa, Samll-Amplitude limit cycles in Liénard systems via multiplicity, J. Differential Equations. 159 (1998), 1015-1039.

[13] A. Gasull, H. Giacomini, J. Llibre, New criteria for the existence and non-existence of limit cycles in Liénard differential systems, Dynamical Systems: An International Journal 24 (2009), 171-185.

[14] M. HAn, H. Yan, J. YANG AND C. LhOtKa, On the number of limit cycles of some Liénard systems, Can. Appl. Math. Q. 17 (2009), 61-83.

[15] M. Han And V.G. Romanovski, On the number of limit cycles of polynomial Liénard systems, Nonlinear Analysis: Real World Applications 14 (2013), 1655-1668.

[16] M. Han, Y. Tian and P. Yu, Small-amplitude limit cycles of polynomial Liénard systems, Science China Math. 56 (2013), doi: 10.1007/s11425-013-4618-9.

[17] J. JiAnd, M. HAN, P. YU AND S. LYNCH, Limit cycles in two types of symmetric Lienard systems, Int. J. Bifurcation and Chaos 17 (2007), 2169-2174.

[18] A. LiÉnARd, Étude des oscillations entrenues, Revue Génerale de l'Électricité 23 (1928), 946-954.

[19] A. Lins, W. De Melo and C.C. Pugh, On Liénard's Equation, Lecture Notes in Math. 597, Springer, Berlin, (1977), pp. 335-357.

[20] C. Li AND J. Llibre, Uniqueness of limit cycles for Liénard differential equations of degree four, J. Differential Equations 252 (2012), 3142-3162.

[21] J. Llibre, Averaging theory and limit cycles for quadratic systems, Rad. Mat. 11 (2002/03), $215-228$.

[22] J. Llibre, A. Makhlouf, Limit cycles of a class of generalized Liénard polynomial equations, preprint, 2013.

[23] J. Llibre, A.C Mereu and M.A. Teixeira, Limit cycles of the generalized polynomial Liénard differential equations, Math. Proc. Camb. Phil. Soc. 148 (2010), 363-383. 
B. GARCÍA, J. LLIBRE AND J.S. PÉREZ DEL RÍO

[24] J. Llibre And C. VAlLS, On the number of limit cycles of a class of polynomial differential systems, Proceedings A of the Royal Society 468 (2012), 2347-2360.

[25] J. Llibre AND C. VALLS, Limit cycles for a generalization of Liénard polynomial differential systems, Chaos, Solitons and Fractals 46 (2013), 65-74.

[26] J. Llibre AND C. VALLS, On the number of limit cycles for a generalization of Liénard polynomial differential systems, Int. J. Bifurcation and Chaos 23 (2013), 1350048-16pp.

[27] N.G. LlOYd, Limit cycles of polynomial systems-some recent developments, London Math. Soc. Lecture Note Ser. 127, Cambridge University Press, 1988, pp. 192-234.

[28] N. G. Lloyd and S. Lynch, Small-amplitude limit cycles of certain Liénard systems, Proc. Royal Soc. London Ser. A 418 (1988), 199-208.

[29] S. LYNCH, Limit cycles if generalized Liénard equations, Applied Math. Letters 8 (1995), $15-17$.

[30] S. LyNCH, Generalized quadratic Liénard equations, Applied Math. Letters 11 (1998), 7-10.

[31] S. Lynch, Generalized cubic Liénard equations, Applied Math. Letters 12 (1999), 1-6.

[32] S. Lynch AND C.J. Christopher, Limit cycles in highly non-linear differential equations, J. Sound Vib. 224 (1999), 505-517.

[33] G.S. Rychkov, The maximum number of limit cycle of the system $\dot{x}=y-a_{1} x^{3}-a_{2} x^{5}, \dot{y}=$ $-x$ is two, Differential'nye Uravneniya 11 (1975), 380-391.

[34] S. Smale, Mathematical Problems for the Next Century, Mathematical Intelligencer 20, (1998), 7-15.

[35] F. Verhulst, Nonlinear Differential Equations and Dynamical Systems, Universitext, Springer, New York, 1991.

[36] J. YAnG, M. Han, V.G. Romanovski, Limit cycle bifurcations of some Liénard systems, J. Math. Anal. Appl. 366 (2010), 242-255.

[37] P. YU AND M. HAN, Limit cycles in generalized Liénard systems, Chaos, Solitons and Fractals 30 (2006), 1048-1068.

Departamento de Matemáticas, Universidad de Oviedo. Avda Calvo Sotelo, s/n., 33007, Oviedo, Spain

E-mail address: belen.garcia@uniovi.es, jspr@uniovi.es

Departament de Matemàtiques, Universitat Autònoma de Barcelona, 08193 Bellaterra, Barcelona, Catalonia, Spain

E-mail address: jllibre@mat.uab.cat 